\title{
Facile Method for Fabricating Superhydrophobic Surface on Magnesium
}

\author{
Munhee Han, Yeonhwa Park, Junewon Hyun, ${ }^{\dagger}$ and Yonghyun Ahn* \\ Department of Chemistry, GRRC, Dankook University, Yongin 448-701, Korea. E-mail: yhahn@dankook.ac.kr \\ ${ }^{\dagger}$ Department of Applied Physics, Dankook University, Yongin 448-701, Korea \\ Received January 7, 2010, Accepted February 8, 2010
}

Key Words: Superhydrophobicity, Wettability, Stearic acid, Chemical etching, Metal deposition

In recent times, technologies based on the imitation of nature have attracted considerable attention. Lotus leaves are known for their self-cleaning effect. The micrometer-scale papillae structure and the epicuticular wax on the lotus leaf contribute to this effect. In a manner similar to the self-cleaning property of lotus leaves, the wettability of solid surfaces is of great interest in daily life and industry. ${ }^{1-4}$ Wettability is controlled by both the geometrical structure of a surface and a low surface energy material coating. A superhydrophobic surface is satisfied with a water contact angle of more than $150^{\circ}$ and a sliding angle of less than $10^{\circ}$. On such a surface, a water drop has a perfectly spherical shape and it easily rolls off and removes deposited contaminants. A superhydrophobic surface thus protects a material from contamination, fogging, and snow deposition.

A material coating with a lower surface energy on a flat surface has a water contact angle of less than $120^{\circ} .5$ This implies that a flat surface will be changed into a rough surface upon impact with the contact interface. Many methods have been reported for constructing micro- and nanostructures on a surface. For example, the sol-gel method, ${ }^{6-8}$ anodization and plasma treatment, ${ }^{9}$ microfabrication with photolithography, ${ }^{10}$ solidification of an alkylketene dimer, ${ }^{11}$ and application of a polymer ${ }^{12-16}$ have been used to obtain a superhydrophobic surface. The presence of micro- and nanometer-scale structures on a surface involves trapping a large amount of air. This causes a reduction in the contact area between water and a solid surface. In addition to the micro- and nanostructures, a material coating with a low surface energy contributes to the superhydrophobicity of the surface. Fluorine-containing compounds have been commonly coated on solid materials in order to fabricate an arti-

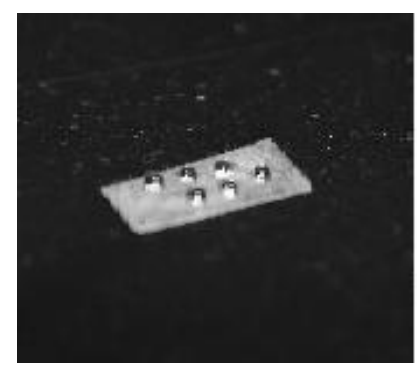

(a)

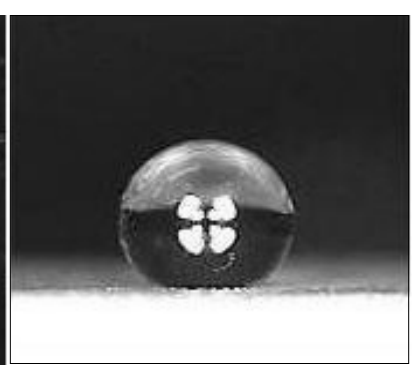

(b)
Figure 1. (a) Optical image of water droplets on Ni deposited Mg surface treated with stearic acid. (b) Profile of water droplet on surface having a contact angle of $144.5^{\circ}$. ficial superhydrophobic surface. ${ }^{17-20}$ Recently, the self-assembly of n-alkanoic acids on a metal surface has been used to prepare a superhydrophobic surface. ${ }^{21-22}$ Au clusters on an ITO glass substrate were modified with n-dodecanethiol to obtain a water contact angle of $173^{\circ} .^{23}$ A series of $n$-alkanoic acids was used to prepare a superhydrophobic surface. Depending on the chain length of the n-alkanonic acids, the contact angle on a flat surface was changed from $68^{\circ}$ to $113^{\circ} .^{24}$ A wet chemical etching surface of an aluminum alloy was treated with stearic acid and $N, N^{\prime}$-dicyclohexylcarbodiimide (DCCD) to obtain a superhydrophobic surface. ${ }^{25}$ Further, the superhydrophobic surface of a polyethyleneimine-coated aluminum wafer was obtained by its reaction with stearic acid and DCCD. ${ }^{26}$ DCCD was used as a dehydration reagent to form either an ester bond or an amide bond.

Most methods for fabricating a superhydrophobic surface are either complicated or require the use of a special instrument. In this study, we have used a chemical deposition process to deposit metal clusters on a magnesium plate and have simply modified the plate with stearic acid.

\section{Experimental Section}

The preparation method was as follows. (1) A piece of magnesium was rinsed in ethanol and then cleaned in distilled water in an ultrasonic bath for $5 \mathrm{~min}$. (2) After drying, the magnesium piece was immersed in $5 \% \mathrm{HCl}$ solution for $2 \mathrm{~min}$, rinsed with distilled water and then dried at $80{ }^{\circ} \mathrm{C}$ for $1 \mathrm{~h}$. (3) The magnesium piece was immersed in a mixed chemical solution $(1.3 \mathrm{~g}$

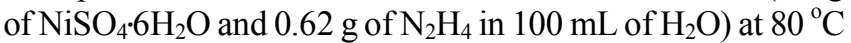
for $4 \mathrm{~h}$ and then dried overnight at room temperature. (4) The magnesium piece was immersed in aqueous stearic acid solution ( $1 \mathrm{~g}$ in $100 \mathrm{~mL}$ of distilled water) for $50 \mathrm{~min}$ and then dried at room temperature for $24 \mathrm{~h}$.

The magnesium surface modified with $5 \% \mathrm{HCl}$ was still a hydrophilic surface with a water contact angle of $20^{\circ}$ (Table 1). A scanning electron microscope (SEM) was used to study the geometrical nano- and microstructures of the superhydrophobic surface.

Fig. 2 shows the typical morphology of a superhydrophobic surface of a Ni-coated magnesium plate. A porous structure and Ni particles were observed on the surface. The metal clusters deposited on the surface exhibited an interesting structure with nanoscale protuberances. The micro- and nanometer-scale structure and cavity on the surface played an important role in trapp- 
Table 1. Water contact angle on surfaces

\begin{tabular}{|c|c|c|c|c|}
\hline Surface & $\begin{array}{l}\text { Untreated } \\
\text { magnesium }\end{array}$ & $\begin{array}{l}\text { Mg surface treated } \\
\text { with } 5 \% \mathrm{HCl}\end{array}$ & $\begin{array}{l}\text { Ni deposited } \\
\text { Mg surface }\end{array}$ & $\begin{array}{l}\mathrm{Ni} \text { deposited Mg surface } \\
\text { treated with stearic acid }\end{array}$ \\
\hline & $\mathrm{CW} ; 58.4^{\circ}$ & $\mathrm{CW} ; 20.4^{\circ}$ & $\mathrm{CW} ; 12.1^{\circ}$ & $\mathrm{CW} ; 144.5^{\circ}$ \\
\hline $\begin{array}{c}\text { Water } \\
\text { contact angle }\left({ }^{\circ}\right)\end{array}$ & & & & \\
\hline
\end{tabular}

(a)

(b)

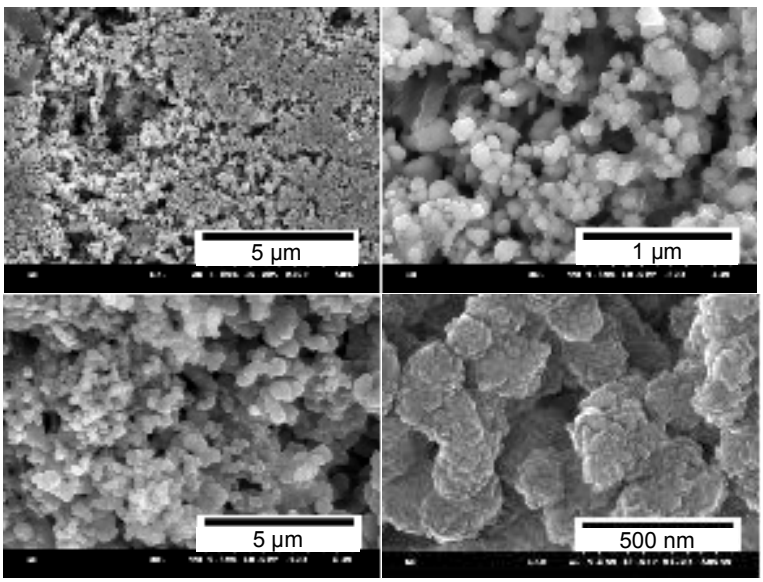

Figure 2. (a) SEM image of Ni deposited on Mg surface. (b) SEM image of Ni deposited on $\mathrm{Mg}$ surface modified with stearic acid.

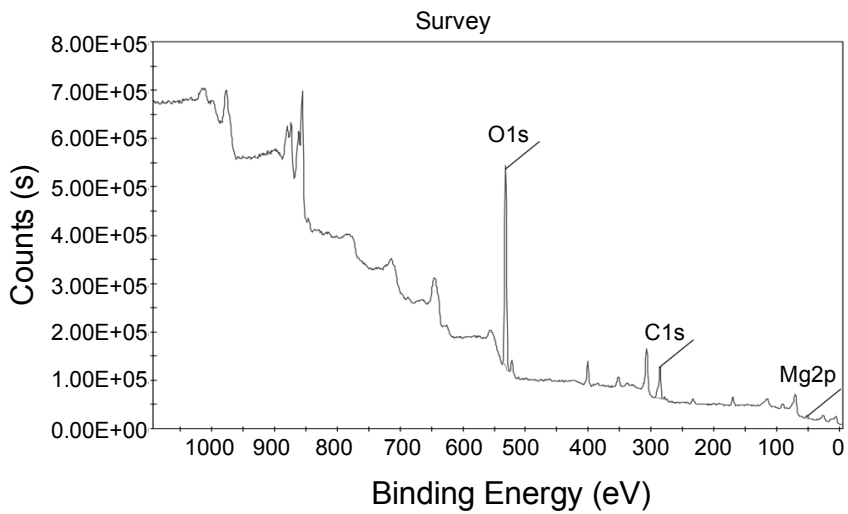

Figure 3. XPS pattern of stearic acid coating on Ni deposited Mg surface.

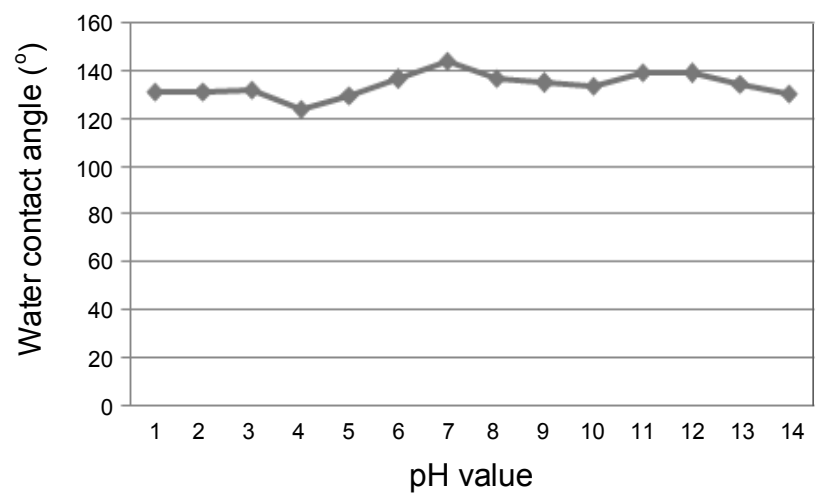

Figure 4. Relationship between $\mathrm{pH}$ and water contact angle on superhydrophobic surfaces of $\mathrm{Mg}$ block. ing air; the trapped air served as a base for water. Low-surfaceenergy materials as well as the rough microstructure of a surface are crucial for fabricating a hydrophobic surface.

XPS signals of $\mathrm{C}$, and $\mathrm{O}$ were observed on the hydrophobic metal surface (Fig. 3). This indicated that the metal surfaces were covered with stearic acid.

Fig. 1 shows water droplets on the Ni-coated magnesium plate modified with stearic acid. Without the stearic acid coating, the water contact angle of the Ni-coated magnesium surface was $12^{\circ}$. The Ni-coated magnesium surface modified with stearic acid was a superhydrophobic surface with a water contact angle of $144.5^{\circ}$. A critical change in the water contact angle was that the self-assembly of stearic acid on the metal clusters largely decreased their surface free energy. There was no obvious change in the roughness and structure after stearic acid treatment (Fig. 2). These results show that the micro- and nanometer-scale structure and the low-surface-energy material are important factors for obtaining superhydrophobic surfaces. Fig. 4 shows the relationship between the $\mathrm{pH}$ and the water contact angle on the superhydrophobic magnesium surface. The contact angles on this surface remained between $124.5^{\circ}$ and $144.5^{\circ}$ with a change in $\mathrm{pH}$ from 1 to 14 . On the basis of the changes observed in the water contact angle of the surface, it was concluded that the $\mathrm{pH}$ of the aqueous solution had a negligible effect on the superhydrophobicity of the metal surface. The superhydrophobic surface was stable over a broad range of $\mathrm{pH}$ values.

\section{Conclusion}

In conclusion, we have developed a simple and inexpensive method for fabricating a superhydrophobic surface of magnesium by metal deposition and stearic acid coating. We fabricated a superhydrophobic surface on magnesium by nickel deposition and surface coating of stearic acid. The fabricated surfaces were stable against acidic and basic solutions.

Acknowledgments. This work was supported by the GRRC program of Gyeonggi province, 66966, Preparation and UV curing characteristics of Silicone Modified Acrylate for Flexible Substrate Hard Coating.

\section{References}

1. Sun, T.; Feng, L.; Gao, X.; Jiang, L. Acc. Chem. Res. 2005, 38, 644. 2. Blossey, R. Nature Materials 2003, 2, 301.

3. Feng, L.; Li, S.; Li, Y.; Li, H.; Zhang, L.; Zhai, J.; Song, Y.; Liu, B.; Jiang, L.; Zhu, D. Adv. Mater. 2002, 14, 1857. 
4. Nakajima, A.; Hashimoto, K.; Watanabe, T. Monatshefte Fur Chemie 2001, 132, 31.

5. Shirtcliffe, N.; Mchale, G.; Newton, M.; Perry, C. Langmuir 2005, $21,937$.

6. Latthe, S.; Imai, H.; Ganesan, V.; Rao, A. Appl. Surf. Sci. 2009, 256,217

7. Lakshmi, R.; Basu, B. J. Colloid and Interface Sci. 2009, 339, 454.

8. Fang, X.; Yu, Z.; Sun, X.; Liu, X.; Qin, F. Front. Chem. Eng. China 2009, 3, 97.

9. Wang, H.; Dai, D.; Wu, X. Appl. Surf. Sci. 2008, 254, 5599.

10. Berendsen, C. W.; Skeren, M.; Najdek, D.; Cerny, F. Appl. Surf. Sci. 2009, 255, 9305.

11. Onda, T.; Shibuichi, S.; Satoh, N.; Tsujii, K. Langmuir 1996, 12, 2125.

12. Levkin, P.; Svec, F.; Frechet, J. Adv. Funct. Mater. 2009, 19, 1.

13. Yuan, Z.; Chen, H.; Tang, J.; Zhao, D. J. Appl. Polymer Sci. 2009, 113,1626 .

14. Feng, L.; Song, Y.; Zhai, J.; Liu, B.; Xu, J.; Jiang, L.; Zhu, D. Angew, Chem. Int. Ed. 2003, 42, 800.
15. Feng, L.; Li, S.; Li, H.; Zhai, J.; Song, Y.; Jiang, L.; Zhu, D. Angew,. Chem. 2002, 114, 1269.

16. Erbil, H.; Demirel, A.; Avcl, Y.; Mert, O. Science 2003, 299, 1377.

17. Chen, L.; Chen, M.; Zhou, H.; Chen, J. Appl. Surf. Sci. 2008, 255, 3459.

18. Guo, Z.; Zhou, F.; Hao, J.; Liu, W. J. Am. Chem. Soc. 2005, 127, 15670 .

19. Kulinich, S.; Farzaneh, M. Surf. Sci. 2004, 573, 379.

20. Nishino, T.; Meguro, M.; Nakamae, K.; Matsushita, M.; Ueda, Y. Langmuir 1999, 15, 4321.

21. Wu, W.; Wang, X.; Liu, X.; Zhou, F. ACS Applied Materials \& Interfaces 2009, 1, 1656.

22. Shustak, G.; Domb, A.; Mandler, D.; Langmuir 2006, $22,5237$.

23. Zhang, X.; Shi, F.; Yu, X.; Liu, H.; Fu, Y.; Wang, Z.; Jiang, L.; Li, X. J. Am. Chem. Soc. 2004, 126, 3064.

24. Shustak, G.; Domb, A.; Mandler, D. Langmuir 2004, 20, 7499.

25. Wang, Q.; Zhang, B.; Qu, M.; Zhang, J.; He, D. Appl. Surf. Sci. 2008, 254, 2009.

26. Ren, S.; Yang, S.; Zhao, Y.; Yu, T.; Xiao, X. Surf. Sci. 2003, 546, 64. 